\title{
Patterns of Depression and Resilience in Children and Adolescents Exposed to an Earthquake: A Latent Profile Analysis
}

\author{
Yue Wang ${ }^{1 *}$, Fenfen $\mathrm{Ge}^{1 *}$, Mentong Wan ${ }^{2}$, and Jun Zhang ${ }^{3 凶}$ \\ ${ }^{1}$ Mental Health Center of West China Hospital, Sichuan University, Chengdu Sichuan, P. R. China \\ ${ }^{2}$ Mentong Wan, Wuyuzhang Honors College, Sichuan University, Chengdu Sichuan, P. R. China \\ ${ }^{3}$ Mental Health Center of West China Hospital and Disaster Medicine Center, Sichuan University, Chengdu Sichuan, P. R. China
}

\begin{abstract}
Objective Depression and resilience are different psychological outcomes caused by experiencing traumatic events. We aimed to 1) explore heterogeneity patterns of co-occurrence between depression symptoms and resilience among children and adolescents exposed to an earthquake and 2) assess covariates (trauma exposure, sex, age, ethnicity, and sleep quality) in identifying the best fitting solution.

Methods Latent profile analysis (LPA) was used to examine patterns of self-reported depression and resilience in an epidemiological sample of 2,887 Chinese youth survivors 1 year after the Lushan earthquake.

Results A suitable 3-class model were identified, which are mild depression/high resilience (65.0\%), severe depression/high resilience (22.1\%), and severe depression/low resilience (12.9\%). Trauma exposure, demographic characteristics and sleep state can be used to identify the different latent classes.

Conclusion Our results contribute to understanding the heterogeneous coexisting patterns of depression and resilience and provide suggestions for identifying high-risk youth survivors and offering effective interventions.

Psychiatry Investig 2021;18(6):580-588
\end{abstract}

Key Words Depression, Resilience, Latent profile analysis, Earthquake, Children and adolescents.

\section{INTRODUCTION}

According to the United Nations International Children Fund, there were about 535 million children and adolescents exposed to traumatic events worldwide in 2016 alone. ${ }^{1}$ Traumatic exposure has been shown to be associated with shortand long-term negative mental health consequences among children and adolescents, ${ }^{2,3}$ and depression is one of the most common stress responses among youth survivors. ${ }^{4-6}$ Considering the following reasons, it's necessary to focus on depression among post-traumatic children and adolescents. Firstly, there is a high prevalence rate of depression (ranging from 7.5\% to $44.8 \%$ ) in this population. ${ }^{7}$ Secondly, depression has some

Received: September 13, 2020 Revised: December 3, 2020

Accepted: March 11, 2021

$\triangle$ Correspondence: Jun Zhang, MD

Mental Health Center of West China Hospital and Disaster Medicine Center, Sichuan University, 28 Dianxinnan Street, Chengdu 610041, China

Tel: +86-18980602251, E-mail: zh2000jun2000@163.com

*These authors contributed equally to this work.

(c) This is an Open Access article distributed under the terms of the Creative Commons Attribution Non-Commercial License (https://creativecommons.org/licenses/by$\mathrm{nc} / 4.0$ ) which permits unrestricted non-commercial use, distribution, and reproduction in any medium, provided the original work is properly cited. negative impacts on both physical (e.g., obesity, pain and cerebrovascular disease) ${ }^{8-10}$ and psychological (e.g., substance abuse/ dependence, major depressive disorder and anxiety disorder) characteristics. ${ }^{11-13}$ Thirdly, an episode of depression in adolescence is associated with a series of mental disorders in adulthood, ${ }^{14-16}$ not only influences the quality of life ${ }^{17}$ but also increases the risk of suicide ${ }^{18}$ and is one of the leading causes of disability globally. ${ }^{19}$

Besides negative psychological outcomes (e.g., depression), traumatic events also can result in some positive psychological changes, such as resilience. ${ }^{20,21}$ Traditionally, psychological resilience was described as an attribute-like personality characteristic, for the reason that some people are overwhelmed by certain hazards while others manage to overcome them. ${ }^{22}$ But now, more and more researchers take resilience as a multidimensional, dynamic capacity ${ }^{23-25}$ which may change over time and under different circumstance. ${ }^{26}$ Specifically, resilience is the ability to recover from traumatic events. ${ }^{27}$ Thus, in this study, we conceptualize resilience as the process of adaptation that follows traumatic events. ${ }^{28-30}$

Thus, what is the relationship between those two different psychological outcomes caused by experiencing traumatic events, 
depression as well as resilience? Previous studies have found that resilience can reduce the risk of depression among individuals with adverse childhood experience, ${ }^{31,32}$ and low resilience to trauma events during adolescence was related to an increased risk of lifelong use of antidepressant. ${ }^{33}$ Although variable-centered approaches had yielded much insight into the relations between depression and resilience, person-centered approaches (latent profile analysis, LPA) have the potential to extend depression and resilience by identifying how different profiles. A person-centered approach allows for distinguishing subpopulations to be identified that differ in the level (quantity) and shape (quality) of the profile indicators, which is not possible with variable-centered analyses. ${ }^{34}$ Furthermore, the person-centered approach contributes to explore the coexisting patterns of depression and resilience among young survivors.

We conducted an LPA study in an epidemiological sample of Chinese youth earthquake survivors. The first aim was to identify the patterns of depression and resilience among children and adolescents exposed to an earthquake; It is well demonstrated that the severity of trauma exposure, ${ }^{35}$ demographic characteristics $^{36,37}$ and sleep quality ${ }^{38,39}$ are associated with depression and/or resilience. Accordingly, the second aim was to investigate whether latent classes with different symptom features could be identified by several factors (e.g. trauma exposure, demographic and sleep quality).

\section{METHODS}

\section{Participants and procedure}

Students were recruited from 21 primary and secondary schools located in Lushan County, Sichuan Province, where was hit by the 2013 Lushan Earthquake (Figure 1). The Lushan earthquake, placed at 7.0 on the Richter scale, occurred on April 20, 2013, resulting in 21 missing, 196 deaths, and 11,470 injured. ${ }^{40}$ The epicenter located in Lushan County, Sichuan, near the Longmengshan Fault, which has the formation with the potential to cause super strong earthquakes. ${ }^{41}$

Data collection was conducted at 1 year after the Lushan earthquake. We used a paper-and-pencil questionnaire and conducted the assessment by well-trained researchers with master's degree in psychology in each class to supervise participants when they filled the questionnaire. Included participants were the students who consented to participate in the survey. Excluded individuals were those who refused to participate or hadn't the ability to complete the investigation. In total, 2,917 children and adolescents agreed to participate. Due to that 33 students hadn't completed the assessments, we finally included 2,887 students in our analysis. The response rate for the assessments was $98.97 \%(2,887 / 2,917)$. Details about sampling strategy showed in Figure 2.

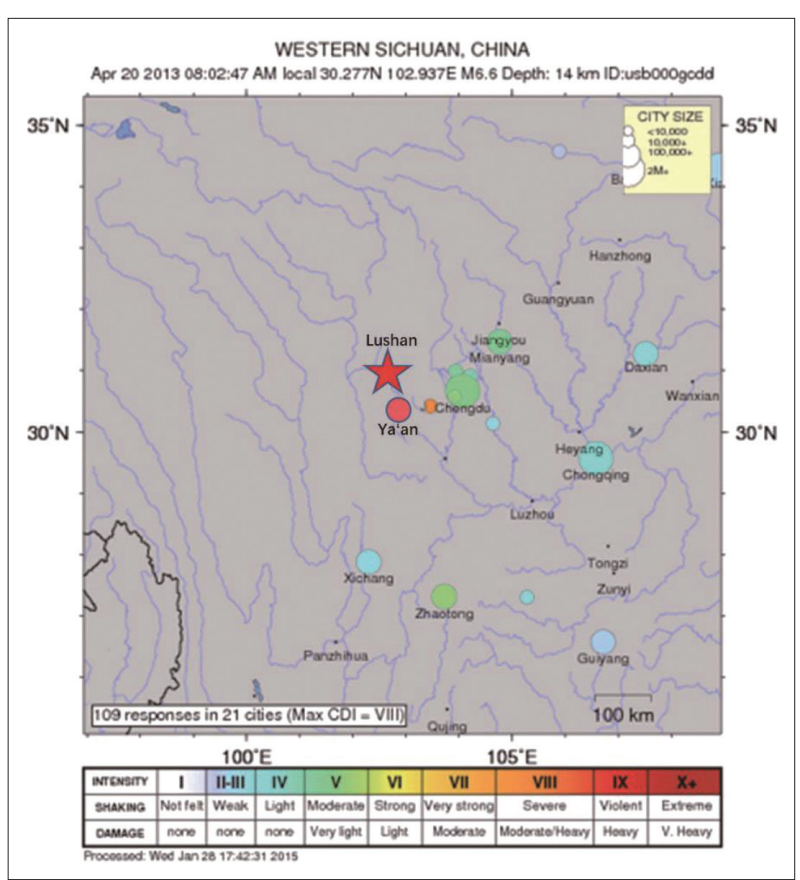

Figure 1. Geographical location of the 2013 Lushan earthquake (red dot), China.

We selected Baoxing County for two reasons: 1) there were 21 primary and secondary schools in this region, satisfying the needs for a large sample; and 2) the directors of the Education Bureau and teachers of these schools paid close attention to the mental health of students. They were willing to sustain this survey. The research protocol was approved by the Ethics Committee of the University of Sichuan and the Education Bureau of Baoxing Country in China and was consistent with the latest version of Helsinki Declaration [IRB approval number: 2017 (103)] and was consistent with the latest vision of Helsinki Declaration. In the present survey, we only attained informed consent from participants and teachers. Because in China, if the local education institution (e.g., the Education Bureau of Baoxing Country) supports a survey as a mental health service to the students, parental consent is not required..$^{42}$ It was also made clear to the students that they could choose whether to participate in the survey or not. ${ }^{40}$ More detailed information was published in a previously paper. ${ }^{43}$

\section{Measures}

Students completed the following self-report questionnaires in their own classroom: sociodemographic characteristics, $\mathrm{Pa}$ tient Health Questionnaire-9 (PHQ-9), Child and Youth Resilience Measure (CYRM), and two self-constructed questionnaires for trauma-exposure and sleep. ${ }^{44}$ Before the investigation was conducted, the purpose and significance of the research were introduced and a psychologist helped interpret some easily confusing items, such as "I solve problems without drugs or 


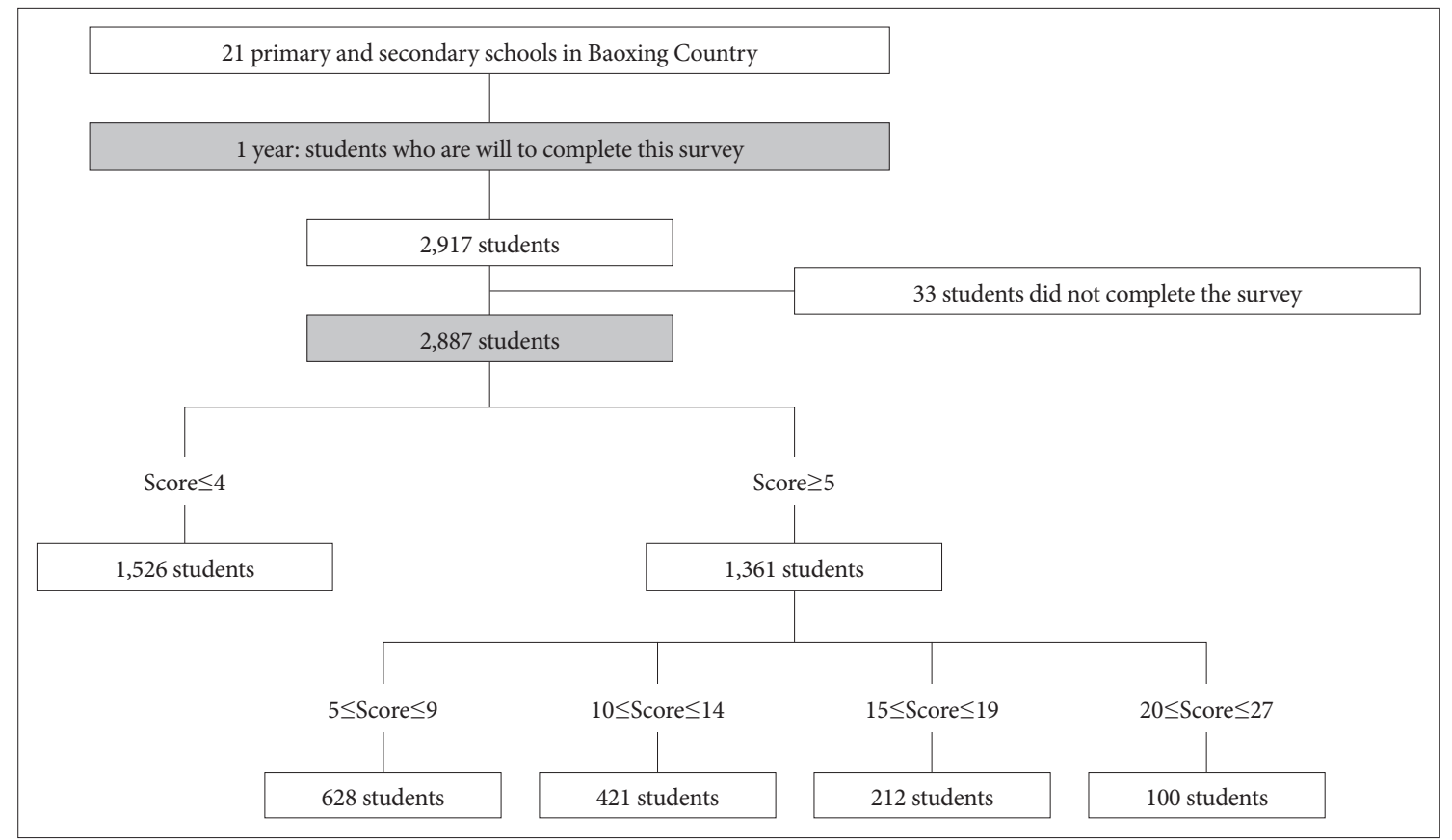

Figure 2. Flow diagram of the participants.

alcohol" and "I try to finish what I start." We provided more detailed information in Supplementary Table 1 (in the onlineonly Data Supplement).

\section{Trauma exposure}

A self-constructed questionnaire included 6 items used to assess trauma exposure severity: 1) Did your immediate family members injured in the earthquake? 2) Did your immediate family members die in the earthquake? 3) Was your house damaged in the disaster? 4) Was there anyone with you after the earthquake? 5) Did the earthquake cause severe property damage to your family? 6) Did you sleep in a tent rather than your own home last night? All answers were coded as binary variables (1 "Yes" or 0 "No"), and we calculated the sum score (range from 0 to 6 ) to reflect the severity of trauma exposure. The total score of the scale was used to assess the severity of the earthquake trauma exposure.

\section{Sleep}

A self-constructed sleep questionnaire was used to assess insomnia and 3 items were included: 1) difficulty falling asleep, 2) difficulty staying asleep, and 3) early waking. All questions and answers were coded as binary variables (yes or no). If the student's answers are all "yes," then this student is considered to have insomnia symptoms.

\section{Patient Health Questionnaire (PHQ-9)}

The self-report symptom scale of the PHQ-9 is a widely used screening tool for depression according to the Diagnostic and
Statistical Manual of Mental Disorder-IV (DSM-IV) criteria. ${ }^{45}$ The scale consists of 9-items and the items are: Over the last two weeks, how often have you been bothered by any of the following problems? 1) Little interest or pleasure in doing things. 2) Felling down, depressed, or hopeless. 3) Trouble falling or staying asleep, or sleeping too much. 4) Feeling tired of having little energy. 5) Poor appetite or overeating. 6) Feeling bad about yourself-or that you are a failure or having let yourself or your family down. 7) Trouble concentrating on things, such as reading the newspaper or watching television. 8) Moving or speaking so slowly that other people could have noticed. Or the opposite-being so fidgety or restless that you have been moving a lot more than usual. 9) Thought that you would be better off dead, or of hurting yourself in some way. The total scores range from 0 to 27 , and can be divided into 4 categories: no depression $(\leq 4)$, minimal (5-9), mild (10-14), moderate (15-19), and severe ( $\geq 20)$. A cut-off point of 10 has been widely accepted for the diagnostic criteria of probable major depressive disorder (pMDD) in different setting. ${ }^{46}$ The Chinese version of PHQ-9 has good reliability and validity ${ }^{47}$ and in the present study, the Cronbach's alpha value was 0.899 .

\section{Child and Youth Resilience Measure (CYRM-12)}

The CYRM-12 is a self-report instrument to measure youth resilience. The specific items are 1) I have people I look up to. 2) Getting an education is important to me. 3) My parent(s)/ caregivers(s) know a lot about me. 4) I try to finish what I start. 5) I solve problems without harming myself or others. 6) I know where to go in my community to get help. 7) I feel I belong(ed) 
to at my school. 8) My family will stand by me during difficult times. 9) My friends stand by me during difficult times. 10) I am treated fairly in my community. 11) I have opportunities to develop skills that will be useful later in life. 12) I enjoy my cultural and family traditions. Items are rated on a 5-point scale from $1=$ does not describe me at all to $5=$ describes me a lot. Higher scores suggest higher levels of resilience. ${ }^{44}$ In the present study, the Cronbach's alpha value was 0.907 , suggesting good reliability.

\section{Data analysis}

Statistical analyses were accomplished by Mplus 7.0 (Linda Muthén \& Bengt Muthén, Los Angeles, CA, USA). The number of missing responses on each item of PHQ-9 were 10, 14, $13,14,23,14,23,14,14,12$, and 17 respectively. As for CYRM12 , they were $55,55,58,70,61,65,61,54,61,63,66$, and 63 , respectively. Missing values were estimated with maximum likelihood procedures. Considering the difference in scale range between PHQ-9 and CYRM-12, all items' scores were converted into standardized Z-scores. The 3-step LPA (R3STEP) approach was conducted via maximum likelihood estimation. ${ }^{48}$ In the first step, the LPA model was conducted based on the PHQ-9 and CYRM-12 scale scores. In the second step, latent class membership was calculated according to the latent class posterior distribution. In the third step, trauma exposure, sex, age, ethnicity, and insomnia were treated as independent variables to predict latent class membership. Odd ratios (ORs) were calculated according to the beta values and standard errors.

The evaluation indexes of LPA model fitting degree are Akaike Information Criteria (AIC) values, Bayesian Information Criteria (BIC) values, adjusted BIC (aBIC), Entropy, Lo-MendellRubin likelihood ratio test (LMR LRT) and Bootstrap-based Likelihood Ratio Test (BLRT). ${ }^{49}$ The smaller values of AIC, BIC and $\mathrm{aBIC}$ are, the better fit of the model is. Entropy is mainly used to evaluate the accuracy of classification, ranging from $0-1$. The closer it is to 1 , the higher accuracy of the classification is. The LMR LRT and BLRT indicators are used to compare the fit differences between the $n$ and n-1 categories. For instance, an LPA model with 4 categories, the p-values for LMR LRT and BLRT are used to compare the differences in fit between the 4 categories and the 3 category models. If the p values of all LMR LRT and BLRT are significant, it means that the four categories of models fit better than the three categories; if the $p$ values of LMR LRT and BLRT are not significant, it means that the three categories of models are better than the four categories. If the $\mathrm{p}$ values of BLRT and LMR LRT are inconsistent, the actual number of potential categories and the number of samples included in the category should be combined to determine the final number of potential categories.

\section{RESULTS}

A total of 2,887 children and adolescents completed the assessment (1,541 female; 2,375 Han-people and 247 meet the criteria of insomnia) (Table 1). The mean score of age was $12.83 \pm 2.58$ and total earthquake exposure was $2.62 \pm 0.77$. Mean scores for the PHQ-9 and CYRM-12 were 6.05 \pm 6.25 and $36.9 \pm 10.86$, respectively. By counting the scores of PHQ-

Table 1. Descriptive information of youth survivors

\begin{tabular}{|c|c|}
\hline Characteristics & Mean \pm SD/N (\%) \\
\hline Age & $12.83 \pm 2.59$ \\
\hline \multicolumn{2}{|l|}{ Sex } \\
\hline Female & $1,541(53.38)$ \\
\hline Male & $1,346(46.62)$ \\
\hline \multicolumn{2}{|l|}{ Ethnicity } \\
\hline Han-people & $2,375(82.26)$ \\
\hline Not Han-people & $512(17.73)$ \\
\hline \multicolumn{2}{|l|}{ Trauma-related experience } \\
\hline $\begin{array}{l}\text { Did your immediate family members injure } \\
\text { in the earthquake? (Yes) }\end{array}$ & $218(7.55)$ \\
\hline $\begin{array}{l}\text { Did your immediate family members die in } \\
\text { the earthquake? (Yes) }\end{array}$ & $174(6.03)$ \\
\hline $\begin{array}{l}\text { Was your house damaged in the disaster? } \\
\text { (Yes) }\end{array}$ & $1,995(69.10)$ \\
\hline $\begin{array}{l}\text { Did the earthquake cause severe property } \\
\text { damage to your family? (Yes) }\end{array}$ & $1,981(68.62)$ \\
\hline $\begin{array}{l}\text { Did you sleep in a tent rather than your own } \\
\text { home last night? (Yes) }\end{array}$ & $528(18.29)$ \\
\hline $\begin{array}{l}\text { Was there anyone with you after the } \\
\text { earthquake? (Yes) }\end{array}$ & $2,678(92.76)$ \\
\hline Total score & $2.62 \pm 0.77$ \\
\hline \multicolumn{2}{|l|}{ Sleep } \\
\hline Difficulty falling asleep & $727(24.93)$ \\
\hline Difficulty staying asleep & $609(21.09)$ \\
\hline Early waking & $1,425(49.36)$ \\
\hline Insomnia & $247(8.56)$ \\
\hline \multicolumn{2}{|l|}{ Depression (PHQ-9) } \\
\hline No depression $(0-4)$ & $1,538(53.27)$ \\
\hline Minimal depression (5-9) & $625(21.65)$ \\
\hline Mild depression (10-14) & $414(14.34)$ \\
\hline Moderate depression (15-19) & $210(7.27)$ \\
\hline Severe depression $(20-27)$ & $100(3.46)$ \\
\hline Total score & $6.05 \pm 6.25$ \\
\hline \multicolumn{2}{|l|}{ Resilience (CYRM-12) } \\
\hline Total score & $36.9 \pm 10.86$ \\
\hline
\end{tabular}

SD: standard deviation, N: number, PHQ-9: Patient Health Questionnaire, CYRM-12: Child and Youth Resilience Measure 
9 that higher than the value of 4 , the probable depression diagnosis is $46.73 \%$ (minimal depression $21.65 \%$, mild depression $14.34 \%$, moderate depression $7.27 \%$ and severe depression $3.46 \%$, respectively).

Fit indicators of the different LPA models are shown in Table 2. The BLRT p-values and LMR p-values were significant for each class category comparison $(\alpha=0.50)$. AIC, BIC, and aBIC tend to keep decreasing as increasing the number of latent classes. Thus, these fit indicators may not help much in choosing suitable models. The 2-class to 5-class model all had acceptable entropy value $(>0.90)$. While, compared with the 3-class model, the 4-class model and the 2-class model showed inappropriate class probability. For the 2-class model, the class probability too simple to reflect symptoms well. For the 4-class model, it has a low proportion of class probability, which is $4.82 \%$. Overall, we choose the 3 -class model as the most suitable model, as it was more meaningful and parsimonious.

The 3-class model was characterized by severe depression/low resilience (12.90\%), severe depression/high resilience (22.11\%), mild depression/high resilience (65.00\%). The 3-class model's profile plot of depression and resilience was shown in Figure 3. In the severe depression/low resilience and mild depression/high resilience subgroups, depression symptoms coexisted with the opposite level of resilience. However, in the severe depression/ high resilience group, individuals presented relatively high levels of depression and relatively high levels of resilience.

The final step of the 3-step LPA results was presented in Table 3. Compared with class-2 (mild depression/high resilience), individuals in class-1 (severe depression/low resilience) and class-3 (severe depression/high resilience) were more likely to be older and suffered insomnia symptom, while individuals in class-2 (mild depression/high resilience) were more likely to be male and not Han-people. Trauma exposure was a significant predictor of the class-3 (severe depression/high resilience) compared with class-1 (severe depression/low resilience) and class-2 (mild depression/high resilience).

Table 2. Fit indices for LPA

\begin{tabular}{crccccccl}
\hline Latent & \multicolumn{1}{c}{$\mathrm{k}$} & \multicolumn{1}{c}{ AIC } & \multicolumn{1}{c}{ BIC } & aBIC & Entropy & LMR & \multicolumn{1}{c}{ BLRT } & \multicolumn{1}{c}{ Class probability (\%) } \\
\hline 1c & 42 & $172,114.973$ & $172,365.628$ & $172,232.179$ & - & - & - & 100 \\
2c & 64 & $159,975.23$ & $160,357.180$ & $160,153.829$ & 0.944 & $<0.0001$ & $<0.0001$ & $23.668 / 76.332$ \\
3c & 86 & $154,435.765$ & $154,949.011$ & $154,675.758$ & 0.934 & 0.0353 & 0.0365 & $12.895 / 22.109 / 64.996$ \\
$4 \mathrm{c}$ & 108 & $150,525.599$ & $151,170.140$ & $150,826.985$ & 0.945 & 0.0164 & 0.0168 & $4.815 / 13.821 / 22.238 / 59.127$ \\
$5 \mathrm{c}$ & 130 & $148,274.685$ & $149,050.522$ & $148,637.465$ & 0.928 & 0.0054 & 0.0057 & $2.840 / 4.780 / 19.120 / 21.060 / 52.200$ \\
\hline
\end{tabular}

AIC: Akaike Information Criteria, BIC: Bayesian Information Criteria, aBIC: adjusted BIC, LMR LRT: Lo-Mendell-Rubin likelihood ratio test, BLRT: Bootstrap-based Likelihood Ratio Test

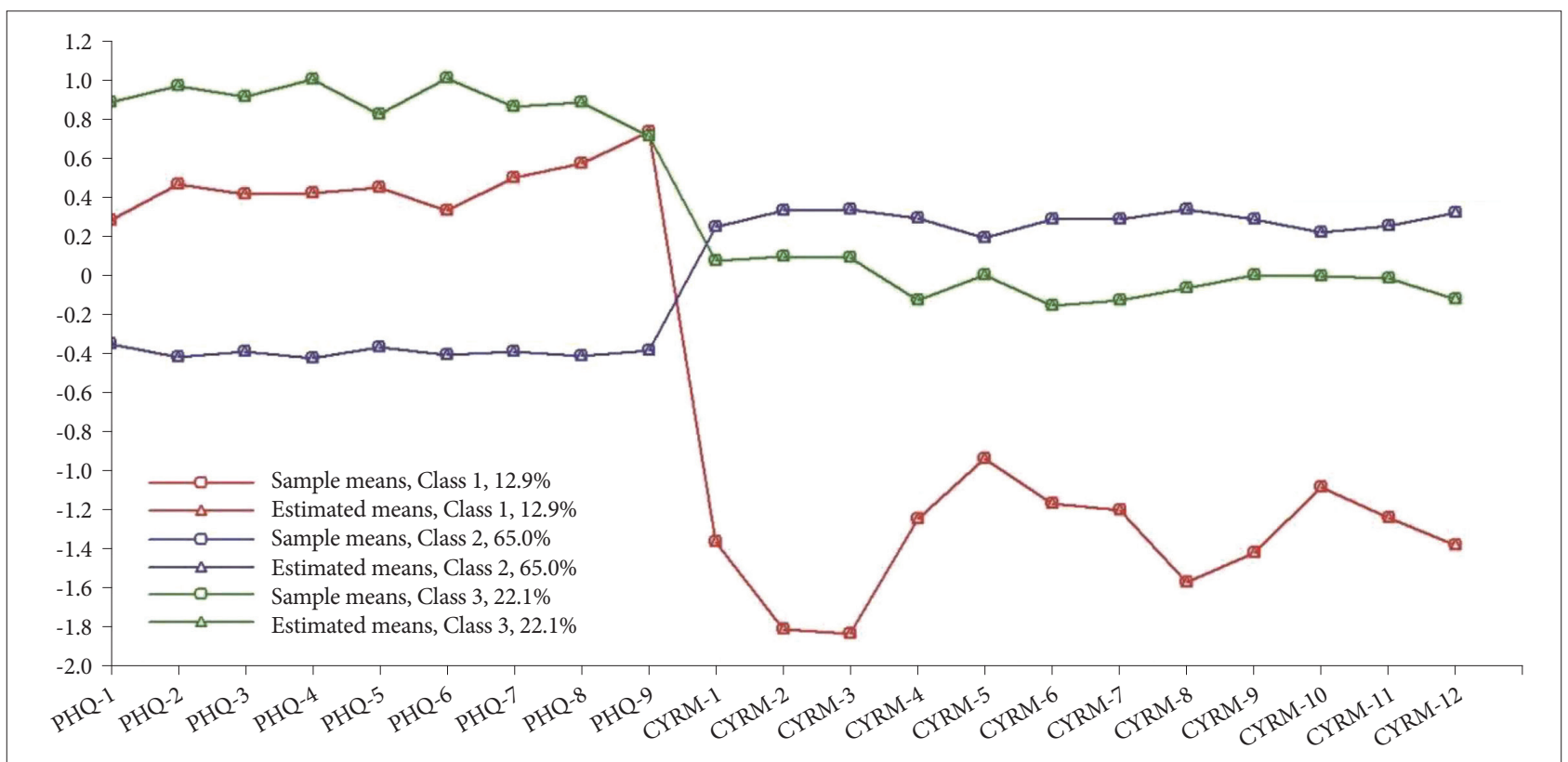

Figure 3. Z-scores of the PHQ-9 and CYRM-12 for the 3-class model symptom profiles. Class-1 severe depression/low resilience; Class-2 mild depression/high resilience; Class-3 severe depression/high resilience. PHQ: Patient Health Questionnaire, CYRM: Child and Youth Resilience Measure. 
Table 3. Factors associated with the latent class groups

\begin{tabular}{|c|c|c|c|c|c|c|c|c|c|c|c|c|}
\hline & \multicolumn{8}{|c|}{ Reference Class 2} & \multirow{2}{*}{\multicolumn{4}{|c|}{$\begin{array}{c}\text { Reference Class } 1 \\
\text { Class } 3\end{array}$}} \\
\hline & \multicolumn{4}{|c|}{ Class 1} & \multicolumn{4}{|c|}{ Class 3} & & & & \\
\hline & B & SE & OR & $\mathrm{p}$ & B & SE & OR & $\mathrm{p}$ & B & SE & OR & $\mathrm{p}$ \\
\hline Sex & -0.283 & 0.119 & 0.754 & 0.018 & 0.019 & 0.100 & 1.019 & 0.852 & 0.301 & 0.141 & 1.351 & 0.033 \\
\hline Age & 0.065 & 0.022 & 1.067 & 0.002 & 0.166 & 0.020 & 1.181 & $<0.001$ & 0.098 & 0.026 & 1.103 & $<0.001$ \\
\hline Ethnicity & -0.777 & 0.201 & 0.460 & $<0.001$ & 0.176 & 0.123 & 1.192 & 0.152 & 0.954 & 0.221 & 2.596 & $<0.001$ \\
\hline Sleep & 1.538 & 0.183 & 4.655 & $<0.001$ & 1.358 & 0.168 & 3.888 & $<0.001$ & -0.180 & 0.186 & 0.835 & 0.333 \\
\hline Trauma exposure & 0.078 & 0.007 & 1.081 & 0.312 & 0.272 & 0.067 & 1.313 & $<0.001$ & 0.194 & 0.092 & 1.214 & 0.034 \\
\hline
\end{tabular}

Class 1: severe depression/low resilience, Class 2: mild depression/high resilience, Class 3: severe depression/high resilience. Sex was code: 0 female, 1 male. Ethnicity was code: 0 Han-people, 1 not Han-people. Sleep: 0 non-insomnia, 1 insomnia

\section{DISCUSSION}

Our study identified 3 latent classes of depression and resilience (severe depression/low resilience, mild depression/high resilience, and severe depression/high resilience) among Chinese youth earthquake survivors, in line with Spahnis founding..$^{50}$ Different latent classes can be identified by different sociodemographic features (e.g., sex, age, and ethnicity), trauma exposure severity and insomnia symptoms.

\section{Three latent class of depression and resilience}

In presented study, a great proportion of individuals were in the mild depression/high resilience group, which was in accordance with findings of Wenchuan earthquake's youth sample. ${ }^{51}$ We both indicated that most individuals obtained positive psychological changes following the earthquake. Based on a previous longitudinal research, resilience does indeed buffer the negative influences of stressful life events over time..$^{52}$ As the positive psychology emphasizes, we should focus on the positive aspects (e.g., resilience and post-traumatic growth) of traumatic experience, not only on the negative side. ${ }^{53,54}$ Our results highlight the potential beneficial influences from the traumatic events.

An interesting phenomenon of this study also sheds light on the controversial relationship between depression and resilience. Negative relationship between those two constructs is shown in the mild depression/high resilience and severe depression/ low resilience subgroups. While, as for the severe depression/ high resilience subgroup, depression and resilience levels were reflective of each other. It's presented a positive association between depression and resilience. These findings indicate complicated reactions among youth survivors, and individual's heterogeneity should be considered.

\section{Factors associated with the latent class groups}

The higher level of trauma exposure was related to the higher probability of being classified into the latent class with a high level of depression (severe depression/high resilience and severe depression/low resilience). A link had been found between trauma exposure and depression, such that individuals who witness the disaster, death/injuries of family members and damage to one's home are being more prone to depression. ${ }^{42,55}$ Meanwhile, other studies also had found that a higher level of trauma exposure was related to a higher level of resilience. When youth survivors have been subject to a traumatic event (such as an earthquake), the experience has led to a positive adjustment. ${ }^{56}$

The role of demographic characteristics was also explored. Compared to their counterparts, male and younger survivors were more likely to enter mild depression/high resilience. Female and male's post-traumatic reactions are markedly different. For example, greater levels of self-esteem and mastery, more common among the male than the female, are positively associated with problem-focused coping, which could reduce the negative impacts of stress. ${ }^{57}$ In contrast, females use more emotion-focused coping strategies, ${ }^{58}$ which may make them at risk of trapping in depression. In addition, females more frequently express fear and shame than do male. ${ }^{59,60}$ Fear and shame can cause survivors to have a negative outlook on their surroundings, namely, low resilience. Low resilience can make negative reappraisals of trauma-related cues and considered the trauma as more threatening. ${ }^{61}$ For youth survivors, they may attain more support from family and society, which are resources related to better mental health. As a type of stress buffer, instrumental aid, active assistance or emotional support from parents or similar others could help to alleviate the impacts of traumatic events. ${ }^{62}$ The previous study also emphasizes the role of supportive relationships with caregivers at preventing children from post-traumatic psychopathology, which could help children dampening the threat processing. ${ }^{63}$

The roles of sleep characteristics were also clarified. The results showed that insomnia individuals were likely to enter the severe depression/low resilience subgroup. Seelig's study found that poor sleep is an important factor and related to the construct of resilience. ${ }^{64}$ Sleep and resilience can share an underly- 
ing neural mechanism and therefore affect one another. Sleep impacted levels of resilience, meaning that greater sleep disturbance could reduce resilience and may potentially predispose individuals to psychopathology such as depression. ${ }^{38}$

\section{Clinical implication}

The current study also provided potential clinical implications. First, based on the person-centered method LPA, our study promotes the understanding of different psychological reactions (depression vs. resilience) as well as individual heterogeneity among youth survivors. Most individuals were in the mild depression/high resilience group, suggesting that resilience may buffer the influence of trauma experience on depression. Thus, in future, we can provide many types of online psychological services for youth survivors to promote their psychological resilience. Second, in our study, we found some important moderating factors (e.g. sleep disturbance) that can influence the relationship between resilience and depression, and indicated more attention should be paid to older insomnia female individuals. As posited by previous research, the presentation of mental disorders are influenced by culture and social milieu, ${ }^{65}$ and Chinese population is more prone to report more somatic symptoms instead of depression symptoms. ${ }^{66}$ Considering sleep disturbance is a relative modifiable factor and more accept mental disorder among Chinese general population. Thus, we can intervene on sleep symptoms to mitigate the outcomes of depressive symptoms. ${ }^{67,68}$ Third, it should be aware that our study was conducted at 1 -year after the earthquake, which assessed the long-term psychological effect of earthquake exposure. In consistent with Sharmass study on the Nepal Earthquake, ${ }^{69}$ we both found a considerable prevalence of depression among youth survivors even after one year of the event. Thus, there is a need for early intervention for youth survivors and we should also pay more attention to the longlasting effects of traumatic event.

\section{Limitations}

Several limitations of our study should be mentioned. First, the findings of our study are based on the self-report scales (e.g., PHQ-9) reported rather than the clinic structure interviewed. And the design of our study was just a cross-sectional study. High quality prospective studies are needed. Second, our research targets on the sample of Chinese youth-earthquake survivors after 1-year exposure, which limits the generalization of the findings. Further studies should examine the profile of depression and resilience in various types of traumatic events among different time points to test the robustness of the model. Third, we only focused on depression in this paper. Other important mental health outcomes (e.g., PTSD, generalized anxiety disorder) are relevant to the trauma events and should not be ignored in further study. Forth, whether resilience is a personality trait or dynamic process is controversial in different studies. In future research, we can evaluate positive psychological through the post-traumatic growth (PTG).

\section{Supplementary Materials}

The online-only Data Supplement is available with this article at https://doi.org/10.30773/pi.2020.0330.

\section{Acknowledgments}

None.

\section{Conflicts of Interest}

The authors have no potential conflicts of interest to disclose.

\section{Author Contributions}

Conceptualization: Jun Zhang. Data curation: Yue Wang. Formal analysis: Fenfen Ge, Mentong Wan. Methodology: Jun Zhang. Software: Yue Wang. Validation: Jun Zhang. Writing_-original draft: Yue Wang, Fenfen Ge. Writing_-review \& editing: Fenfen Ge, Mentong Wan, Jun Zhang.

\section{ORCID iDs}

Yue Wang

Fenfen Ge

Mengtong Wan

Jun Zhang https://orcid.org/0000-0002-0349-5681

https://orcid.org/0000-0003-1516-6698

https://orcid.org/0000-0002-3115-4331

https://orcid.org/0000-0001-6358-681X

\section{REFERENCES}

1. Cerna-Turoff I, Fischer HT, Mayhew S, Devries K. Violence against children and natural disasters: a systematic review and meta-analysis of quantitative evidence. PLoS One 2019;14:e0217719.

2. Oral R, Ramirez M, Coohey C, Nakada S, Walz A, Kuntz A, et al. Adverse childhood experiences and trauma informed care: the future of health care. Pediatr Res 2016;79:227-233.

3. Dube A, Moffatt M, Davison C, Bartels S. Health outcomes for children in Haiti since the 2010 earthquake: a systematic review. Prehosp Disaster Med 2018;33:77-88.

4. Cenat JM, Derivois D. Long-term outcomes among child and adolescent survivors of the 2010 Haitian earthquake. Depress Anxiety 2015;32: 57-63.

5. Blanc J, Rahill GJ, Laconi S, Mouchenik Y. Religious beliefs, PTSD, depression and resilience in survivors of the 2010 Haiti earthquake. J Affect Disord 2016;190:697-703.

6. Derivois D, Cenat JM, Joseph NE, Karray A, Chahraoui K. Prevalence and determinants of post-traumatic stress disorder, anxiety and depression symptoms in street children survivors of the 2010 earthquake in Haiti, four years after. Child Abuse Negl 2017;67:174-181.

7. Tang BH, Liu X, Liu Y, Xue C, Zhang LL. A meta-analysis of risk factors for depression in adults and children after natural disasters. BMC Public Health 2014;14:623.

8. Hasler G, Pine DS, Kleinbaum DG, Gamma A, Luckenbaugh D, Ajdacic V, et al. Depressive symptoms during childhood and adult obesity: the Zurich Cohort Study. Mol Psychiatry 2005;10:842-850.

9. Kleiber B, Jain S, Trivedi MH. Depression and pain: implications for symptomatic presentation and pharmacological treatments. Psychiatry (Edgmont) 2005;2:12-8.

10. Robinson RG, Morris PL, Fedoroff JP. Depression and cerebrovascular disease. J Clin Psychiatry 1990;51(Suppl):26-31; discussion 32-33.

11. McLeod GF, Horwood LJ, Fergusson DM. Adolescent depression, adult mental health and psychosocial outcomes at 30 and 35 years. Psychol Med 2016;46:1401-1412. 
12. Gilchrist EEA, Sadler DW. The role of depression in unnatural death: a case-based retrospective study. J Affect Disord 2019;259:7-14.

13. Dindo LN, Recober A, Haddad R, Calarge CA. Comorbidity of migraine, major depressive disorder, and generalized anxiety disorder in adolescents and young adults. Int J Behav Med 2017;24:528-534.

14. Giaconia RM, Reinherz HZ, Paradis AD, Hauf AMC, Stashwick CK. Major depression and drug disorders in adolescence: general and specific impairments in early adulthood. J Am Acad Child Adolesc Psychiatry 2001;40:1426-1433.

15. Copeland WE, Shanahan L, Costello EJ, Angold A. Childhood and adolescent psychiatric disorders as predictors of young adult disorders. Arch Gen Psychiatry 2009;66:764-772.

16. Dierker L, Rose J, Selya A, Piasecki TM, Hedeker D, Mermelstein R. Depression and nicotine dependence from adolescence to young adulthood. Addict Behav 2015;41:124-128.

17. Schnurr PP, Lunney CA, Bovin MJ, Marx BP. Clinical. Posttraumatic stress disorder and quality of life: extension of findings to veterans of the wars in Iraq and Afghanistan. Psychol Rev 2009;29:727-735.

18. Korczak DJ, Goldstein BI. Childhood onset major depressive disorder: course of illness and psychiatric comorbidity in a community sample. J Pediatr 2009;155:118-123.

19. Gore FM, Bloem PJN, Patton GC, Ferguson J, Joseph V, Coffey C, et al. Global burden of disease in young people aged 10-24 years: a systematic analysis. Lancet 2011;377:2093-2102.

20. Faye C, McGowan JC, Denny CA, David DJ. Neurobiological mechanisms of stress resilience and implications for the aged population. Curr Neuropharmacol 2018;16:234-270.

21. Southwick SM, Vythilingam M, Charney DS. The psychobiology of depression and resilience to stress: implications for prevention and treatment. Annu Rev Clin Psychol 2005;1:255-291.

22. Michael RMD. Psychosocial resilience and protective mechanisms. Am J Orthopsychiatry 2010;57:316-331.

23. Laird KT, Krause B, Funes C, Lavretsky H. Psychobiological factors of resilience and depression in late life. Transl Psychiatry 2019;9:88.

24. De Terte I, Stephens C. Psychological resilience of workers in high-risk occupations. Stress Health 2014;30:353-355.

25. Robertson IT, Cooper CL, Sarkar M, Curran T. Resilience training in the workplace from 2003 to 2014: a systematic review. J Occup Organ Psychol 2015;88:533-562.

26. Smith GC, Hayslip Jr B. Resilience in adulthood and later life: What does it mean and where are we heading? Annu Rev Gerontol Geriatr 2012;32:3-28.

27. Norris FH, Tracy M, Galea S. Looking for resilience: understanding the longitudinal trajectories of responses to stress. Soc Sci Med 2009;68: 2190-2198.

28. Masten AS. Resilience in developing systems: progress and promise as the fourth wave rises. Dev Psychopathol 2007;19:921-930.

29. Aburn G, Gott M, Hoare K. What is resilience? An integrative review of the empirical literature. J Adv Nurs 2016;72:980-1000.

30. Fletcher D, Sarkar M. Psychological resilience a review and critique of definitions, concepts, and theory. Eur Psychol 2013;18:12-23.

31. Poole JC, Dobson KS, Pusch D. Childhood adversity and adult depression: the protective role of psychological resilience. Child Abuse Neglect 2017;64:89-100.

32. Schulz A, Becker M, Van der Auwera S, Barnow S, Appel K, Mahler J, et al. The impact of childhood trauma on depression: does resilience matter? Population-based results from the Study of Health in Pomerania. J Psychosom Res 2014;77:97-103.

33. Howell KH, Miller-Graff LE, Schaefer LM, Scrafford KE. Relational resilience as a potential mediator between adverse childhood experiences and prenatal depression. J Health Psychol 2020;25:545-557.

34. Gabriel AS, Daniels MA, Diefendorff JM, Greguras GJ. Emotional labor actors: a latent profile analysis of emotional labor strategies. J Appl Psychol 2015;100:863-879.

35. Wang Y, Xu J, Lu Y. Associations among trauma exposure, post-trau- matic stress disorder, and depression symptoms in adolescent survivors of the 2013 Lushan earthquake. J Affect Disord 2020;264:407-413.

36. Wellman CL, Bangasser DA, Bollinger JL, Coutellier L, Logrip ML, Moench KM, et al. Sex differences in risk and resilience: stress effects on the neural substrates of emotion and motivation. J Neurosci 2018; 38:9423-9432.

37. Nam CR, Lee DH, Lee JY, Choi AR, Chung SJ, Kim DJ, et al. The role of resilience in internet addiction among adolescents between sexes: a moderated mediation model. J Clin Med 2018;7:222.

38. Chatburn A, Coussens S, Kohler MJ. Resiliency as a mediator of the impact of sleep on child and adolescent behavior. Nat Sci Sleep 2013; 6:1-9.

39. van der Zwan JE, de Vente W, Tolvanen M, Karlsson H, Buil JM, Koot $\mathrm{HM}$, et al. Longitudinal associations between sleep and anxiety during pregnancy, and the moderating effect of resilience, using parallel process latent growth curve models. Sleep Med 2017;40:63-68.

40. Zhang Y, Zhang J, Zhu S, Du C, Zhang W. Prevalence of probable posttraumatic stress disorder and determination of its relationship with everyday functioning in children and adolescents by using generalized estimating equations. Asia Pac Psychiatry 2017;9:e12262.

41. Cause Analysis of the M8.0 Wenchuan Earthquake. 2008. Available at: https://www.cea.gov.cn/manage/html/8a8587881632fa5c0116674a018 300cf/_content/08_05/30/1212119940937.html.) Accessed on Jan 26, 2021.

42. Ying L, Wu X, Lin C, Jiang L. Traumatic severity and trait resilience as predictors of posttraumatic stress disorder and depressive symptoms among adolescent survivors of the Wenchuan earthquake. PLoS One 2014;9:e89401.

43. Ge F, Yuan M, Li Y, Zhang J, Zhang W. Changes in the network structure of posttraumatic stress disorder symptoms at different time points among youth survivors: a network analysis. J Affect Disord 2019;259: 288-295.

44. Liebenberg L, Ungar M, LeBlanc JC. The CYRM-12: a brief measure of resilience. Can J Public Health 2013;104:e131-e135.

45. Kroenke K, Spitzer RL, Williams JB. The PHQ-9: validity of a brief depression severity measure. J Gen Intern Med 2001;16:606-613.

46. Moriarty AS, Gilbody S, McMillan D, Manea L. Screening and case finding for major depressive disorder using the Patient Health Questionnaire (PHQ-9): a meta-analysis. Gen Hosp Psychiatry 2015;37: 567-576.

47. He XY, Li CB, Qian J, Cui HS, Wu WY. Reliability and validity of a generalized anxiety disorder scale in general hospital outpatient. Shanghai Arch Psychiatry 2010;22:200-203.

48. Auxiliary Variables in Mixture Modeling: A 3-Step Approach using Mplus. Mplus Web Notes: No. 1. http://statmodel.com/examples/webnotesAuxMixture_submitted. 2013.

49. Morin AJS, Morizot J, Boudrias JS, Madore I. A Multifoci person-centered perspective on workplace affective commitment: a latent profile/ factor mixture analysis. Organ Res Methods 2011;14:58-90.

50. Spahni S, Morselli D, Perrig-Chiello P, Bennett KM. Patterns of psychological adaptation to spousal bereavement in old age. Gerontology 2015;61:456-468.

51. Cao C, Wang L, Wu J, Li G, Fang R, Cao X, et al. Patterns of posttraumatic stress disorder symptoms and posttraumatic growth in an epidemiological sample of Chinese earthquake survivors: a latent profile analysis. Front Psychol 2018;9:1549.

52. Sheerin CM, Lind MJ, Brown EA, Gardner CO, Kendler KS, Amstadter $\mathrm{AB}$. The impact of resilience and subsequent stressful life events on MDD and GAD. Depress Anxiety 2018;35:140-147.

53. Kobau R, Seligman MEP, Peterson C, Diener E, Zack MM, Chapman $\mathrm{D}$, et al. Mental health promotion in public health: perspectives and strategies from positive psychology. Am J Public Health 2011;101:e1e9.

54. Tedeschi RG, Calhoun LG. Posttraumatic growth: conceptual foundations and empirical evidence. Psychol Inq 2004;15:1-18. 
55. Goenjian AK, Walling D, Steinberg AM, Roussos A, Goenjian HA, Pynoos RS. Depression and PTSD symptoms among bereaved adolescents 61/2 years after the 1988 spitak earthquake. J Affect Disord 2009; 112:81-84.

56. Osorio C, Probert T, Jones E, Young AH, Robbins I. Adapting to stress: understanding the neurobiology of resilience. Behav Med 2017;43: 307-322.

57. Rosenfield S, Mouzon D. Gender and Mental Health. In: Aneshensel CS, Phelan JC, Bierman A, Editors. Handbook of the Sociology of Mental Health. Dordrecht: Springer Netherlands, 2013, p.277-296.

58. Vingerhoets AJ, Van Heck GL. Gender, coping and psychosomatic symptoms. Psychol Med 1990;20:125-135.

59. Simon RW. Contributions of the Sociology of Mental Health for Understanding the Social Antecedents, Social Regulation, and Social Distribution of Emotion. In: Avison WR, McLeod JD, Pescosolido BA, Editors. Mental Health, Social Mirror. Boston, MA: Springer US, 2007, p.239-274.

60. Else-Quest NM, Higgins A, Allison C, Morton LC. Gender differences in self-conscious emotional experience: a meta-analysis. Psychol Bull 2012;138:947-981.

61. Florian V, Mikulincer M, Taubman O. Does hardiness contribute to mental health during a stressful real-life situation? The roles of appraisal and coping. J Pers Soc Psychol 1995;68:687-695.

62. Thoits PA. Mechanisms linking social ties and support to physical and mental health. J Health Soc Behav 2011;52:145-161.

63. Schaefer LM, Howell KH, Schwartz LE, Bottomley JS, Crossnine CB. A concurrent examination of protective factors associated with resilience and posttraumatic growth following childhood victimization. Child Abuse Negl 2018;85:17-27.

64. Seelig AD, Jacobson IG, Donoho CJ, Trone DW, Crum-Cianflone NF, Balkin TJ. Sleep and health resilience metrics in a large military cohort. Sleep 2016;39:1111-1120.

65. Gureje O, Lewis-Fernandez R, Hall BJ, Reed GM. Systematic inclusion of culture-related information in ICD-11. World Psychiatry 2019;18: 357-358.

66. Parker G, Gladstone G, Chee KT. Depression in the planet's largest ethnic group: the Chinese. Am J Psychiatry 2001;158:857-864.

67. McCall WV, Blocker JN, D’Agostino R Jr, Kimball J, Boggs N, Lasater B, et al. Treatment of insomnia in depressed insomniacs: effects on health-related quality of life, objective and self-reported sleep, and depression. J Clin Sleep Med 2010;6:322-329.

68. Manber R, Edinger JD, Gress JL, San Pedro-Salcedo MG, Kuo TF, Kalista T. Cognitive behavioral therapy for insomnia enhances depression outcome in patients with comorbid major depressive disorder and insomnia. Sleep 2008;31:489-495.

69. Sharma A, Kar N. Posttraumatic stress, depression, and coping following the 2015 Nepal earthquake: a study on adolescents. Disaster Med Public Health Prep 2019;13:236-242. 


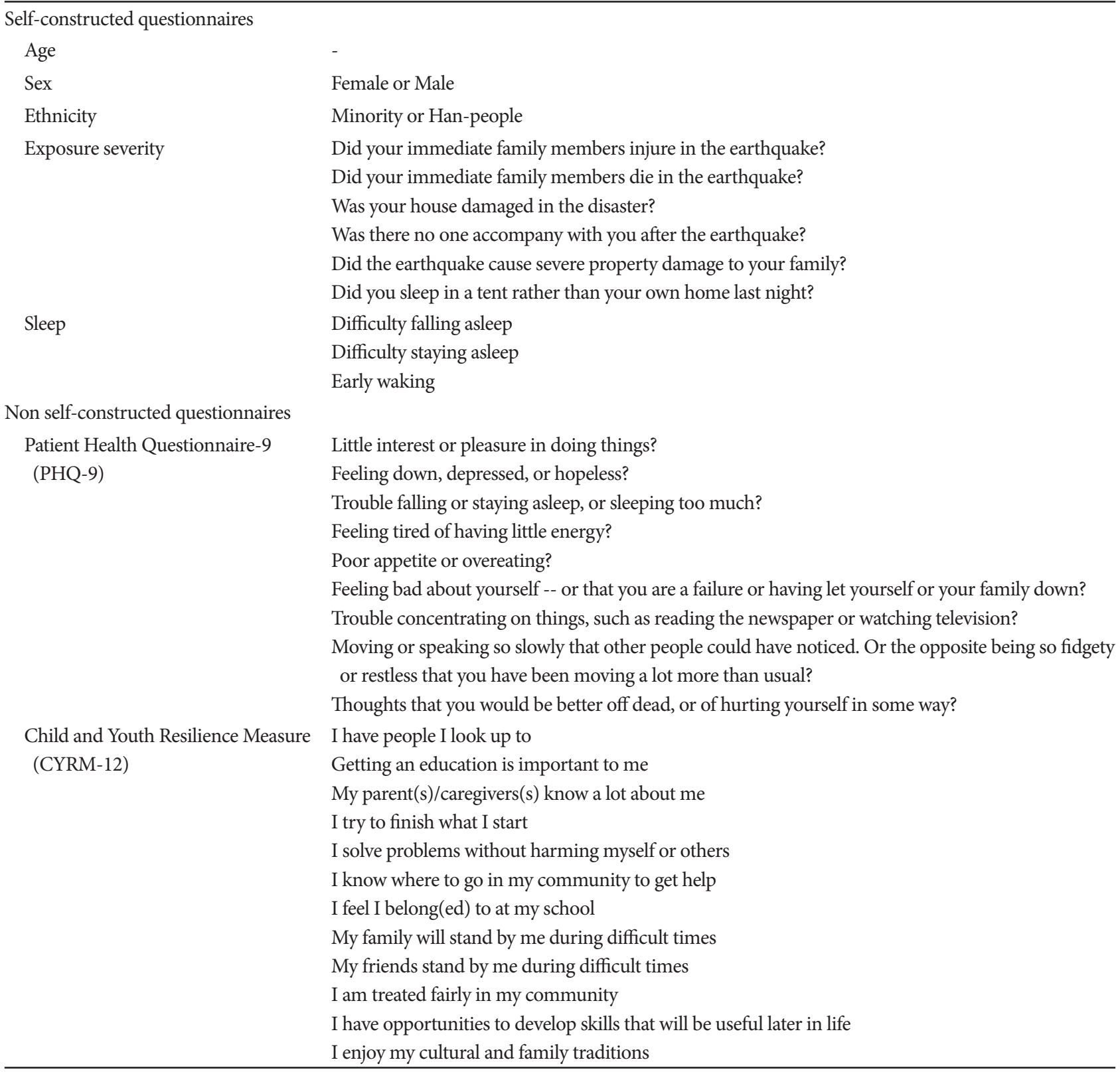

